\title{
Role of CT Mastoids in the Diagnosis and Surgical Management of Chronic Inflammatory Ear Diseases
}

\author{
Juveria Majeed $^{1,2} \cdot$ L. Sudarshan Reddy ${ }^{1,2}$
}

Received: 4 March 2016/Accepted: 3 October 2016/Published online: 13 October 2016

(C) Association of Otolaryngologists of India 2016

\begin{abstract}
Chronic Suppurative Otitis Media (CSOM) is a chronic inflammation of middle ear cleft. It presents with discharging ear and decreased hearing. The diagnosis is mostly on clinical examination with otoscope or oto endoscope. Computerised Tomography (CT) of mastoids is done to evaluate the extent of the disease and its complication. High resolution CT is now the investigation of choice for temporal bone disease. This study emphasizes on the importance of CT scan in diagnosis of inflammatory ear diseases and most importantly identifying pitfalls or complications which a surgeon can come across during surgery. The aims and objectives of this study is (1) to establish the efficacy of CT in the diagnosis of the complications and surgical management of chronic inflammatory pathologies of middle ear. (2) To find subgroups of CSOM where CT is particularly useful. In this series, a total of 25 cases presenting to our OPD at Govt. ENT Hospital between 2013 and 2014 have been diagnosed and findings of surgery correlated with HRCT scan of temporal bones done preoperatively. In this study, $64 \%$ of the patients were male and incidence of CSOM with patients undergoing surgery belonged to the age group 21-30 years (32\%). The most common presenting symptom was ear discharge (92\%) and decreased hearing (96\%). The most common type of pathology in this study was attic perforation $(36 \%)$ and granulations $(40 \%)$ followed by cholesteatoma $(36 \%)$ and mucosal edema $(16 \%)$. Not all
\end{abstract}

L. Sudarshan Reddy

drlsudarshanreddy9@gmail.com

1 Govt. Medical College/Hospital, Nizamabad, Telangana, India

2 G-4, Jamuna Sadan, Mayuri Marg, Begumpet, Hyderabad, Telangana 500016, India cases presented with complications, facial palsy (12\%) and mastoid abscess ( $8 \%$ ) were among few complications seen. 14 patients $(56 \%)$ of 25 cases underwent simple cortical mastoidectomy followed by 9 cases $(36 \%)$ for modified radical mastoidectomy and atticotomy for 2 cases $(8 \%)$. CT scan findings correlated well with surgical findings for cholesteatoma, middle ear mass and bone erosions. Where as for ossicular integrity and facial canal dehiscence, there was a discrepancy.

Keywords Computerised tomography - Temporal bone . Cholesteatoma - Ossicular integrity · Granulations .

Facial canal dehiscence

\section{Introduction}

Chronic Otitis Media is a chronic inflammation within the mucosa of the middle ear cleft with varying degrees of edema, submucosal fibrosis, hypervascularity and infiltration with lymphocytes, macrophages and plasma cells. Middle ear cleft include the eustachian tube, hypotympanum, mesotympanum, epitympanum, aditus and mastoid air cell system [1]. There are two types of CSOM- tubotympanic and atticoantral. To know the severity and extent of the disease and to know anatomical variations or destruction/erosions and thence to avoid any complications during surgery, a CT scan of the temporal bone is must to proceed any further with the diagnosis and management of ear diseases.

The CT system was invented in 1972 by Godfrey Newbold Hounsfield of EMI Central Research Laboratories using X-rays. Allan McLeod Cormack of Tufts University independently invented the same process and they shared a Nobel Prize in medicine in 1979 [2]. There are 4 
generations of CT scanners. Modern multi-detector, multirow CT systems can complete a scan of the chest, for example, in less time than it takes for a single breath hold and display the computed images in near real time. Images that used to take hours to acquire and days to process are now accomplished in seconds. The number of cross sectional images that can be produced has increased from about a dozen to many hundreds [3].

\section{Patients and Methods}

Patients with suspected middle ear disease attending our outpatient department of ENT Govt. ENT Hospital, Hyderabad from 2013 to 2014 were included in the study group (Tables 1, 2, 3, 4, 5, 6, 7, 8).

Sample size: a total no. of 25 patients have been included in the study.

Type of study: prospective study.

Inclusion criteria:

A. Atticoantral type of disease.

B. Any age with unsafe type of disease, both adults and children.

C. Both males and females.

D. Patients with CSOM complications both intra and extracranial complications.

Table 1 Sex distribution

\begin{tabular}{llcc}
\hline S. no. & Sex & No. of cases & Percentage \\
\hline 1 & Male & 16 & 64 \\
2 & Female & 9 & 36 \\
3 & Total & 25 & 100 \\
\hline
\end{tabular}

Table 2 Age distribution

\begin{tabular}{lllll}
\hline S. no. & Age (years) & No. of cases & Total no. of cases & Percentage \\
\hline 1 & $0-10$ & 04 & 25 & 16 \\
2 & $11-20$ & 05 & 25 & 20 \\
3 & $21-30$ & 08 & 25 & 32 \\
4 & $31-40$ & 05 & 25 & 20 \\
5 & $41-50$ & 01 & 25 & 4 \\
6 & $51-60$ & 02 & 25 & 8 \\
\hline
\end{tabular}

Total no. of cases 25

Table 3 Incidence of CSOM in relation to side

\begin{tabular}{llll}
\hline S. no. & Side of ear & No. of cases & Percentage \\
\hline 1 & Right & 11 & 44 \\
2 & Left & 14 & 56
\end{tabular}

Total no. of cases 25
Table 4 Incidence of symptoms

\begin{tabular}{llcc}
\hline S. no. & Presenting complaints & No. of cases & Percentage \\
\hline 1 & Discharge & 23 & 92 \\
2 & Decreased hearing & 24 & 96 \\
3 & Mass in EAC & 2 & 8 \\
4 & Giddiness & 1 & 4 \\
5 & Ringing sensation in ears & 19 & 76 \\
6 & Pain & 4 & 16 \\
7 & Postaural abscess & 2 & 8 \\
8 & Facial palsy & 3 & 12 \\
\hline
\end{tabular}

Total no. of cases 25

Table 5 Type of tympanic membrane perforation

\begin{tabular}{llll}
\hline S. no. & Perforation & No. of cases & Percentage \\
\hline 1 & Attic & 09 & 36 \\
2 & Central OR subtotal & 05 & 20 \\
3 & Marginal & 03 & 12 \\
4 & Retraction pockets & 08 & 32 \\
\hline
\end{tabular}

Table 6 Middle ear pathology

\begin{tabular}{llcc}
\hline S. no. & Pathology & No. of cases & Percentage \\
\hline 1 & Granulation & 10 & 40 \\
2 & Cholesteatoma & 9 & 36 \\
3 & Polyp & 2 & 8 \\
4 & Mucosal edema & 4 & 16 \\
\hline
\end{tabular}

Table 7 Complications of CSOM

\begin{tabular}{llll}
\hline S. no. & Complication & No. of cases & Percentage \\
\hline 1 & Facial palsy & 03 & 12 \\
2 & Labyrinthitis & 01 & 4 \\
3 & Mastoid abscess & 02 & 8 \\
4 & Sub dural effusion & - & - \\
5 & Temporal lobe abscess & - & - \\
6 & Sinus thrombosis & 01 & 4 \\
7 & Total & 07 & 28 \\
\hline
\end{tabular}

Table 8 Type of surgery

\begin{tabular}{llll}
\hline $\begin{array}{l}\text { S. } \\
\text { no. }\end{array}$ & Surgery & $\begin{array}{l}\text { No. of } \\
\text { cases }\end{array}$ & Percentage \\
\hline 1 & Simple mastoidectomy & 14 & 56 \\
2 & Modified radical mastoidectomy & 09 & 36 \\
3 & Atticotomy with simple & 02 & 8 \\
$\quad$ mastoidectomy & Nil & Nil \\
\hline
\end{tabular}


E. Patients giving consent to undergo CT scan and surgery.

Exclusion criteria:

Patients excluded were:
A. Tubotympanic type of CSOM.
B. Revision cases.
C. Bilaterality of disease.

\section{Methodology}

25 All patients included in the study had been explained about the study, need for them to undergo CT Mastoids preoperatively and need for surgery and follow up. Patients were selected randomly initially, then included or excluded based on the criteria listed above. Both males, females and children were included in the study done. Lowest age being 7 years in my study.

All patients included first underwent otoendoscopy to rule out tubotympanic type of chronic ear diseases and also to exclude other pathologies which causes discharging ear like otitis externa and otomycosis. Patients with active squamosal type or atticoantral type were included after confirming the findings in endoscopy of the ear.

Consent for undergoing CT scan and followed by appropriate surgery were taken in written. All basic hematological examinations done for surgical fitness of the patients.

All patients were subjected to CT Scan of temporal bone, high resolution in both axial and coronal settings (supine and prone axis) with $1.5 \mathrm{~mm}$ thick slices. Scanning commenced from the lower margins of external auditory meatus and extend upwards from the arcuate eminence of the superior semicircular canal as seen on the lateral tomogram. Slight extension of the head was given to avoid the gantry tilt and thereby protect the lens from radiations. Coronal images were obtained perpendicular to the axial plane from the cochlea to the posterior semicircular canal. A radiologist was assigned to read and report all these scans.

Surgeries according to the pathology were undertaken, mostly under local anaesthesia, nine patients underwent GA mostly because of their age. All 25 patients underwent mastoidectomies, postaural approach and graft used was temporalis fascia in all. Simple cortical mastoidectomies in 14 patients, modified radical mastoidectomies in 9 patients and atticotomy in 2 patients. On table, the surgical findings were noted in a proforma made exclusively to note down all surgical and radiological findings. These surgical findings were correlated to the radiological findings seen preoperatively on CT mastoids. The surgical findings were also informed to the radiologist to improve the learning curve. Patients were followed up to success rates of surgeries performed with time to time and regular follow ups until ear becomes dry.

\section{Observations and Results}

This study of 25 patients is based on the observations made in the cases of chronic suppurative otitis media treated in the Department of ENT and HEAD \& NECK Surgery, Govt.ENT Hospital, Hyderabad.

Otorrhea and decreased hearing been most common complaints.

Simple Cortical Mastoidectomy was done in most cases, 14 cases, $56 \%$. In case of cholesteatoma extending the middle ear cavity, attic and antrum was present, MRM is the best surgery. Hence in all 9 cases of cholesteatoma, MRM is done. MRM (canal wall down) technique gives good view to remove all the disease, it gives good postoperative hearing, and it reduces the chances of residual disease (Tables 9, 10, 11).

\section{In Comparison to CT Scan}

CT findings correlated very well with surgical findings $(100 \%)$ in cases of cholesteatoma. All cases reported as cholesteatoma in CT were confirmed at surgery (Table 12, 13, 14, 15).

Cholesteatoma was identified on CT scan film by

Table 9 Follow up records

\begin{tabular}{llll}
\hline S. no. & Follow up & No. of cases & Percentage \\
\hline 1 & Regular & 21 & 84 \\
2 & Irregular & 4 & 16 \\
3 & Not turned-up & - & - \\
\hline
\end{tabular}

Total no. of cases 25

Table 10 Results after 3 months

\begin{tabular}{llll}
\hline S. no. & Follow up & No. of cases & Percentage \\
\hline 1 & Healed cavities & 21 & 84 \\
2 & Discharging & 4 & 32 \\
\hline
\end{tabular}

Total no. of cases 25

Table 11 Cholesteatoma

\begin{tabular}{lll}
\hline S. no. & Cholesteatoma & No. of cases \\
\hline 1 & ON CT & 9 \\
2 & At surgery & 9 \\
\hline
\end{tabular}

Total no. of cases 25 
Table 12 Middle ear mass

\begin{tabular}{lll}
\hline S. no. & Middle ear mass & No. of cases \\
\hline 1 & IN CT scan & 15 \\
2 & IN Surgery & 15 \\
\hline
\end{tabular}

Total no. of cases 25

Table 13 Bone erosion

\begin{tabular}{lll}
\hline S. no. & Bone erosion & No. of cases \\
\hline 1 & IN CT scan & 3 \\
2 & IN Surgery & 3 \\
\hline
\end{tabular}

Total no. of cases 25

Table 14 Facial nerve dehiscence

\begin{tabular}{lll}
\hline S. no. & FN dehiscence & No. of cases \\
\hline 1 & In CT & 2 \\
2 & In surgery & 3 \\
\hline
\end{tabular}

Total no. of cases 25

Table 15 Ossicular chain integrity

\begin{tabular}{lll}
\hline S. no. & Integrity of ossicular chain & No. of cases \\
\hline 1 & IN CT scan & 16 \\
2 & IN Surgery & 11 \\
\hline
\end{tabular}

Total no. of cases 25

1. Evidence of soft tissue density.

2. Low attenuation.

3. Absence of enhancement on contrast.

4. Presence of erosion of bone.

The above table shows that the CT findings of soft tissue density i.e. middle ear mass (polyp, granulations) correlated very well with the surgical findings $(100 \%)$.

Mass may be hypertrophied mucosa or granulations in the middle ear.

These can be identified by

1. Abnormal soft tissue density.

2. Enhancement on contrast.

3. Absence of bony erosion.

All reported cases were confirmed at surgery.

Bone erosion is early radiological sign of cholesteatoma. Above table shows that there is $100 \%$ correlation between radiological and surgical findings in case of detecting bony erosions.

All cases reported in CT were conformed at surgery.

Cases reported as facial nerve dehiscence in CT scan were two, whereas on surgery there were three facial nerve canal dehiscence. Facial nerve dehiscence was better seen in coronal cuts of CT. Overlying soft tissues cause a loss of contrast gradience.

In CT Scan Malleus and body of incus are visualized easily. Stapes and I.S. joint are visualized with difficulty. The status of ossicular chain was confirmed in 11 out of 16 cases in this study $(68.75 \%)$.

\section{Discussion}

Chronic Inflammatory ear diseases can be safe/tubotympanic or unsafe/atticoantral type of diseases. Or can be said as active or inactive mucosal or squamous type. Safe type of disease with a dry perforation in pars tensa generally, but not always causes any complication. But with pathology in the attic area like granulations and cholesteatoma, only clinical examination is not enough to rule out underlying pathology in temporal bone, which not documented before can lead untoward consequences. Hence radiological investigation becomes necessary to assess the pathology in the middle ear cleft. It also becomes necessary in those patients whose tympanic membrane is not visible due to external ear conditions. It is especially useful in children who do not cooperate or in whom visualization of tympanic membrane is difficult. It becomes imminent to investigate radiologically in the patients with intracranial complications. In the present study, all such patients were subjected to $\mathrm{CT}$ scan of mastoid bones.

HRCT of Mastoids has a capability of displaying all the anatomical structures demonstrated by conventional tomography plus many other structures by virtue of projectional advantage and superior contrast resolution $[4,5]$. This contrast resolution permits the demonstration of soft tissue masses, which is frequently not possible with conventional tomography. Hence, CT imaging is an accurate method of depicting the extent \& nature of disease in CSOM.

The increased information about disease extent provided by routine $\mathrm{CT}$ imaging in all patients with CSOM would assist in-patient counselling, planning the surgical approach and prepares the surgeon for difficult situations.

In the present study we observed

i. The presence and extent of abnormal soft tissue opacity.

ii. Status of the ossicular chain.

iii. Erosion of tegmen or dura.

iv. Facial nerve dehiscence.

v. Fistula in semi circular canal.

During surgery, operative findings are noted.

In the present study, the youngest patient was 7 years and the eldest was 53 years old. Eight patients (32\%) were in the age group of $21-30$ years, five patients $(20 \%)$ each 
in the age groups of 11-20 and 31-40 years. Studies from other countries shows an average age about 35.1 years as in Paperella and Kim [6]. This variation is due to greater incidence of CSOM cases in children in our country. Male:female ratio was $1.77: 1$, more for males in accordance with the study done by Vlastarakos et al. [7] $98 \%$ of the patients belonged to low socioeconomic class leading to poor nutrition and lack of hygiene (less in developed countries) [8].

The most common presenting symptom was otorrhea (92\%) and hearing loss (96\%) followed by tinnitus $(76 \%)$. Left ear discharge in $56 \%$ and Right ear discharge in $44 \%$ as shown.

Facial palsy was seen in three cases, mastoid abscess in two cases and labyrinthitis and sigmoid sinus thrombosis one case each.

Tympanic membrane examination showed attic perforation in 9 cases $(36 \%)$. Subtotal perforation in 5 cases (20\%). Marginal perforation in 3 cases (12\%). Retraction pockets seen in 8 cases (32\%). Polyps obscuring view of tympanic membrane was seen in two cases. Middle ear pathology included cholesteatoma in nine cases, polyps in two cases, granulations in ten cases and mucosal edema in 4 cases as shown.

Fifty-six percentage of the patients underwent simple cortical mastoidectomy, $36 \%$ underwent modified radical mastoidectomy and $8 \%$ underwent atticotomy with simple mastoidectomy. MRM is an effective method to manage cholesteatoma in a single staged procedure [9]. Cases those had cholesteatoma underwent MRM for complete clearance.

Mastoid cavities healed well after regular follow ups in $84 \%$ of the patients. Correlating the radiological and surgical findings- Mastoid bone were sclerotic in all 25 cases. CT scan has $100 \%$ sensitivity and specificity to know the type of mastoid pneumatisation.

\section{Cholesteatoma}

In the present study, CT has $100 \%$ sensitivity and $90 \%$ specificity for diagnosing cholesteatoma through CT images which is in correlation with the studies of Sirigiri and Dwaraknath [10] 0.18 It has less sensitivity for cholesteatoma in antrum and aditus where as $100 \%$ sensitivity for cholesteatomas in epitympanum and hypotympanum.

Cholesteatoma was identified on CT scan film by

1. Evidence of soft tissue density.

2. Low attenuation.

3. Absence of enhancement on contrast.

4. Presence of erosion of bone.

All cases reported as cholesteatoma in CT were conformed at surgery. The appearance of abnormal soft tissue opacity associated with bone erosion is highly suggestive of cholesteatoma and absence of erosion mostly excludes the cholesteatoma. The purpose of scanning is to know the extent of the lesion.

The diagnosis of cholesteatoma in CT is based on the identification of a sharply demarcated soft tissue mass in the middle ear and bony destruction. Inflammatory diseases of middle ear except cholesteatoma was made out by the absence of erosion of otic capsule or ossicular chain, correlated with the studies done by Johnson et al. [11]. Johnson et al. [11] - found that the presence of a welldefined edge to the soft tissue mass and erosion of otic capsule or ossicular chain were a sure indication of a Cholesteatoma.

A high false positive and false negative rate in pre operative CT scan was reported in one of the studies and it was suggested that detection rate was dependent on the anatomical site of the disease. This study has achieved $75 \%$ results.

It was suggested in one study that the ability of MRI in detecting soft tissue changes in cholesteatoma is superior to the CT scan. This study has achieved $90 \%$ results.

In the present study using the criteria stated above for the diagnosis of Cholesteatoma an accurate assessment could be made out in $100 \%$ of the cases. However the suggestion given by B. J. O'Reilly that the diagnosis of Cholesteatoma on CT especially in patients with intact tympanic membrane as in Congenital Cholesteatoma and Combined Approach Tympanoplasty and residual disease in the sinus tympani and the facial recess is valuable.

\section{Soft Tissue Mass}

Present study: HRCT was $84 \%$ sensitive and $88.8 \%$ specific in identifying soft tissue mass. Mafee et al. [12] and O'Reilly et al. [13] have similar results, whereas Jackler et al. [14] and Garber and Dort [15] found it to be less sensitive and specific. However, HRCT is less sensitive in differentiating cholesteatoma from granulations. It was suggested that $\mathrm{CT}$ scans were able to detect soft tissue masses in the mastoid and middle ear in almost all cases, although it was believed that it was possible to identify cholesteatoma by its characteristically low attenuation values. Cholesteatoma was present in 80 of the cases explored when bone erosion was present in association with soft tissue density on CT.

\section{Bone Erosion}

Bone erosion is early radiological sign of cholesteatoma. Present study: All cases (100\%) reported in CT were conformed at surgery. HRCT detected scutum erosion 
accurately in all cases. Hence, HRCT is $100 \%$ sensitive and specific to detect scutum erosion as per this study. This is in accordance to study by Rocher et al. [16]. (1995) but contrasts with study by Vlastarakos et al. [7], where no correlation was found.

Best seen in axial sections; the tegmen slopes downward anteriorly and is not well visualized in coronal sections. Erosion of the posterior wall of the mastoid and the sinus plate occur in extensive Cholesteatomas and may lead to septic Thrombophlebitis of the lateral sinus and cerebellar abscess formation. O'Reilly et al. [13]—detected tegmen erosion in 5 out of 11 cases there was false positives, mainly used axial scans to detect the bony erosion. They stated that it is not possible to reliably demonstrate a dehiscence in the tegmen on axial cuts alone, but even on using coronal cuts they found the effect of partial volume averaging giving rise the false impression of a defect.

HRCT detects bone erosion in the middle ear cleft which includes scutum, tegmen tympani, tegmen antri, sinus plate and outer cortex of the mastoid process. Pre operative information of bony erosion gives the surgeon a head on for planning the surgery.

\section{Sigmoid Sinus Thrombosis}

In the present study, there was only 1 patient which showed sinus plate erosion. Sample size of the study being small, the results were not in accordance with any study. The patient presented with vomiting, giddiness and an episode of seizure. On CT mastoid, there was soft tissue attenuation indicating cholesteatoma.

\section{Facial Canal Dehiscence}

Facial nerve canal can be eroded by cholesteatoma, the facial nerve dysfunction occurs in approximately $1 \%$ of patients with cholesteatoma. Most facial nerve dehisences occur at horizontal part and this part was clearly seen on coronal cuts. Mastoid segment erosions are best seen in sagittal and coronal sections. However sometimes overlying soft tissues cause a loss of contrast gradience resulting in difficulty to comment on the facial nerve condition. Facial nerve dehiscence was better seen in coronal cuts of CT. Overlying soft tissues cause a loss of contrast gradience.

In the present study-CSOM with facial nerve palsy was seen in $0.08 \%$ cases. Where as CT showed facial canal dehiscence in only $0.12 \%$ patients. This is much less than incidence seen by Magliulo et al. [17] in their study where it was $27 \%$.

Jackler (1984), Mafee et al. [12] and O'Reilly [13]—in many cases reported that loss of contrast gradience due to overlying soft tissue obscured a small dehiscence in facial canal.
Banerjee et al. (2003) - are of the opinion that Radio surgical correlation is poor in relation to Facial nerve assessment on CT study.

\section{Ossicular Erosion}

Ossicular erosion was seen in $64 \%$ of the cases recorded in this study. Incus was the most commonest ossicle to be involved. Incus was seen to be eroded in almost all cases of cholesteatoma [9], followed by stapes and malleus, in accordance with the findings of Keskin et al. [18]. In CT Scan Malleus and body of incus are visualized easily. Stapes and I.S. joint are visualized with difficulty. Most of the middle ear pathologies appear as soft tissue attenuation on HRCT $[19,20]$, resulting in non- visualisation of the ossicles.

In one of the studies, the radiological predictions were compared with the operative findings in each case. This form of imaging (CT) proved to be highly accurate in depicting the extent of soft tissue within the middle ear cleft and mastoid. With the exception of the long process of the incus and the stapes superstructure, the state of the ossicular chain was correctly predicted in over $90 \%$ of cases. Erosion of the labyrinth was clearly depicted in 4 of the 5 cases in which it occurred. A correct pathological diagnosis was made radiologically in $88 \%$ cases. The selective use of this modality in the evaluation of patients with chronic suppurative otitis media is valuable.

Jackler et al. [14] —were able to predict the state of ossicular destruction in only seven percent of cases. One study doubted that CT could demonstrate the ossicular chain reliably because of the combination of partial volume averaging and tissue silhouetting. Fifty percentage results were achieved in this study.

\section{Congenital Cholesteatoma}

In the present study, 2 cases of congenital cholesteatoma are included out of 25 cases $(8 \%)$. Congenital cholesteatoma is difficult to differentiate from acquired type, but clinical features may help; this is commonly seen in children with intact tympanic membrane and absence of previous otologic disease. In our study, one case was of 7 year old female and another 52 year old presenting at first with generalized symptoms of sinus thrombosis. On HRCT, a well circumscribed soft tissue mass seen in antrum and sigmoid sinus plate erosion seen. Congenital cholesteatoma appears as well-marginated expansile hypodense lesion. Differentiating them by imaging alone is difficult, but history and molecular biological techniques are helpful in differentiating them [21].

Appropriate projections for ossicles and various middle ear structures: The human temporal bone is an extremely 
complex structure. CT scanning has proved to be the diagnostic imaging method of choice for studying the normal and pathologic details of the temporal bone [21].

CT of the temporal bone should always include at least two projections [22]. The use of a single projection may lead to serious mistakes, since structures parallel to the plane of section are seen only partially or not at all. The basic projection is of course the direct axial (horizontal) plane, since this is the most suitable and practical as well as the easiest projection to obtain for the baseline study of the temporal bone [5, 23-25]. Direct coronal sections can be obtained with many scanners; however, direct sagittal sections are hard or impossible to obtain because of the limitations of the CT scanners. O'Reilly et al. [13]. In his study, it is stated that axial scans are more satisfactory as they depict LSSC in its entirety and are less likely to produce false positives. Nevertheless, useful information can also be obtained from coronal scans and hence both sections should be employed. In acute mastoid infection CT scan shows, diffuse opacification of the middle ear and mastoid air cells without evidence of bone resorption. In cholesteatoma the CT appearance is lateral attic wall erosion. The extra dural abscess can occur when pus collects between the dura and bone, which becomes later thickened and covered with granulation tissue. This thickening is seen on a CT as an irregular enhancement.

A few minutes discussion with the radiologists about the images demonstrating the course of the facial nerve, the relationship of the inner ear and the condition of the ossicles can be of great help in pre operative counseling and also helps in avoiding the hazards during surgery. The same information discussed with the radiologist improves his/her learning curve.

\section{Conclusions and Summary}

- The present study was carried out in the Department of ENT, Govt. ENT Hospital, Osmania Medical College.

- 25 patients are included in this prospective type of study.

- The following conclusions can be reliably reached by means of this study.

- Ideally all cases should be scanned in both axial and coronal planes and sometimes even sagittal view as some structures are viewed best in their appropriate projections.

- The presence and distribution of soft tissue in the middle ear cleft and mastoid could confidently be predicted using this modality.

- Indeed, it was observed, that a scan showing no evidence of soft tissue essentially excluded the presence of a cholesteatoma.
- Soft tissue density in the Middle Ear cleft could be because of granulation tissue or edematous middle ear mucosa. Differentiating point between soft tissue density from cholesteatoma and other pathologies like granulation tissue or polyp is by the presence of bony erosions and loss of ossicular integrity.

- The malleus, body and short process of incus are well visualized. However, the long process of incus and the stapes suprastructure cannot be reliably imaged on these scans. Thus, visualizing the entire ossicular chain was satisfactory. In ossicular erosion, incus was most commonly involved followed by malleus and stapes.

- All the mastoids were sclerotic in this study and well demonstrated in CT.

- The visualisation of thin bony structures (facial nerve canal, tegmen, LSCC) may be misleading due to errors in computer reconstruction of their images and overlying soft tissues cause a loss of contrast gradience, still it is possible to detect facial nerve dehiscence and defects in tegmen tympani in significant number of cases.

- CT findings are inconsistent with surgical findings in terms of facial canal dehiscence and status of ossicular integrity due to soft tissue opacity, which can be because of granulations and mucosal edema also.

- The sinus tympani area is extremely well appreciated in axial cuts, and evaluation of the sinus tympani prior to surgery can help the surgeon to avoid injury to the facial nerve while doing surgery in this area.

- In conclusion, its known that CSOM can at times be life threatening and warrants otolaryngologists to be familiar with the standard techniques for these patients.

- Advent of HRCT and improvements in radiological technique has definitely improved study of the temporal bone in patients with CSOM, which includes evaluation of the extent and sites of involvement and interrelationships of the tympanomastoid compartment with adjacent neurovascular structures.

- CT scan should not be seen as indispensable but rather, as a useful aid to management. HRCT offers information of extent of the disease process, about the vital structures and helps to plan the type of surgery.

- Hence this study emphasizes on the use of CT can be recommended not only in cases suspected with potential complications but also in all cases of COM to know the extent of disease, varied pneumatization and the presence of anatomical variations, which should alert the clinician and guide in surgical approach and treatment plan.

- This study concludes that use of CT Mastoids is to be encouraged by otolaryngologists because only a skilled, aware and alert surgeon is the key to successful diagnosis and treatment of CSOM. 
Funding This study is author's independent work. No funds taken.

\section{Compliance with Ethical Standards}

Conflict of interest The authors declare that they have no conflict of interest.

Human and Animal Rights Animals were not involved in this study.

Ethical Approval All procedures performed in studies involving human participants were in accordance with the ethical standards of the institutional and with the 1964 Helsinki declaration and its later amendments or comparable ethical standards.

Informed Consent Informed consent was obtained from all individual participants included in the study.

\section{References}

1. Browning GG, Merchant SN, Kelly G, Swan LRC, Canter R, McKerrow WS (2008) Chronic otitis media. In: Kerr AG (ed) Scott-Brown's otolaryngology, chap 237c, 7th edn, vol 3. Arnold, London, pp 3395-3445

2. Som PM, Curtin HD (2003) Head and neck imaging, 4th edn, vol 2. Mosby, St. Louis

3. Drake R, Vogl AW, Mitchell AWM (2014) Gray's Anatomy. Descriptive and applied anatomy of adult temporal bone. Elsevier, Churchill

4. Ballantyne J (ed) (1978) Operative surgery-EAR, 3rd edn. Butterworth, London

5. Shambaugh JR (1990) Surgery of the ear, surgical management of CSOM, 4th edn. WB Saunders and Co.

6. Paparella MM, Kim CS (1977) Mastoidectomy update. Laryngoscope 1977(87):88

7. Vlastarakos PV, Kiprouli C, Pappas S, Xenelis J, Maragoudakis P, Troupis G et al (2012) CT scan versus surgery: how reliable is the pre-operative radiological assessment in patients with chronic otitis media. Eur Arch Otorhinolaryngol 269:81-86

8. Bluestone CD (1998) Epidemiology and pathogenesis of chronic suppurative otitis media: implications for prevention and treatment. Int J Pediatr Otorhinolaryngol 42:207

9. Kennedy K, Vrabec J, Quinn Jr. FB (1999) Cholesteatoma: pathogenesis and surgical management. Department of Otolaryngology, UTMB, Grand Rounds Presentation
10. Sirigiri RR, Dwaraknath K (2011) Correlative study of HRCT in attico-antral disease. Indian $\mathrm{J}$ Otolaryngol Head Neck Surg 63:155-158

11. Johnson DW, Voorhees RL, Lufkin RB, Hanafee W, Canalis R (1983) Cholesteatomas of the temporal bone: role of computed tomography. RSNA Radiol Soc N Am 148(3)

12. Mafee MF, Levin BC, Applebaum EL, Campos M, James CF (1988) Cholesteatoma of the middle ear and mastoid A comparison of CT scan and operative findings. Otolaryngol Clin N Am 21:265-293

13. O'Reilly BJ, Chevretton EB, Wylie I, Thakkar C, Butler P, Sathanathan N et al (1991) The value of CT scanning in chronic suppurative otitis media. J Laryngol Otol 105:990-994

14. Jackler RK, Dillon WP, Schindler RA (1984) Computed tomography in suppurative ear disease: a correlation of surgical and radiographic findings. Laryngoscope 94:746-752

15. Garber LZ, Dort JC (1994) Cholesteatoma: diagnosis and staging by CT scan. J Otolaryngol 23:121-124

16. Rocher P, Carlier R, Attal P, Doyon D, Bobin S (1995) Contribution and role of the scanner in the pre-operative evaluation of chronic otitis. Radiosurgical correlation apropos of 85 cases. Ann Otolaryngol Chir Cervicofac 112:317-323

17. Magliulo G, Colicchio MG, Appiani MC (2011) Facial nerve dehiscence and cholesteatoma. Ann Otol Rhinol Laryngol 120:261-267

18. Keskin S, Cetin H, Töre HG (2011) The correlation of temporal bone CT with surgery findings in evaluation of chronic inflammatory diseases of the middle ear. Eur J Gen Med 8:24-30

19. Trojanowska A, Drop A, Trojanowski P, Rosiñska Bogusiewicz K, Klatka J, Bobek-Billewicz B (2012) External and middle ear diseases: radiological diagnosis based on clinical signs and symptoms. Insights Imaging 3:33-48

20. Lemmerling MM, De Foer B, VandeVyver V, Vercruysse JP, Verstraete KL (2008) Imaging of the opacified middle ear. Eur J Radiol 66:363-371

21. Arangasamy A, Chandrasekaran K, Balakrishnan S (2012) Soft tissue attenuation in middle ear on HRCT. In: Mini-Symposia Head and Neck, vol 22, no. 4, pp 298-304

22. Mahmood FM et al (1998) Direct sagittal CT in the evaluation of temporal bone disease. AJNR Am J Neuroradiol 9:371-378

23. Valvassori GE, Mafee MF (1985) The temporal bone. In: Carter BL (ed) Computed tomography of the head and neck. Churchill Livingstone, New York, p 171

24. Zonneveld FW (1983) The value of non-reconstructive multiplanar CT for the evaluation of the petrous bone. Neuroradiology 25:1-10

25. Zonneveld FW, Van Waes PFG, Damsma P, Rabischong P, Vignaud J (1983) Direct multiplanar computed tomography of the petrous bone. Radiographies 3:41 